\title{
A CONSTRUCTION OF ASYMPTOTIC SOLUTIONS AND THE EXISTENCE OF SMOOTH NULL-SOLUTIONS FOR A CLASS OF NON-FUCHSIAN PARTIAL DIFFERENTIAL OPERATORS
}

\author{
TAKESHI MANDAI
}

\section{§1. Introduction}

Consider a partial differential operator

$$
P=\sum_{j+|\alpha| \leq m} a_{j, \alpha}(t, x) \partial_{t}^{\jmath} \partial_{x}^{\alpha}, \quad a_{m, 0}(t, x) \equiv t^{x},
$$

where $\kappa$ is a non-negative integer and $a_{j, \alpha}$ are real-analytic in a neighborhood of $(0,0) \in \mathbf{R}_{t} \times \mathbf{R}_{x}^{n}$.

M. S. Baouendi and C. Goulaouic [1] defined Fuchsian partial differential operators, and proved the unique solvability of the characteristic Cauchy problems in the category of real-analytic (or holomorphic) functions, which is a generalization of the classical Cauchy-Kowalevsky theorem. They also proved a generalization of the Holmgren uniqueness theorem. Especially, from their results it easily follows that if $P$ is a Fuchsian operator with real-analytic coefficients, then there exist no sufficiently smooth null-solutions. Here, a Schwartz distribution $u$ in a neighborhood of $(0,0)$ is called a null-solution for $P$ at $(0,0)$, if $P u=0$ in a neighborhood of $(0,0)$ and $(0,0) \in \operatorname{supp} u \subset\{t \geq 0\}$, where supp $u$ denotes the support of $u$.

The author considered the characteristic Cauchy problems for a class of operators wider than the Fuchsian operators in [3]. In that result, he showed the unique solvability of the characteristic Cauchy problems in the category of functions which are of class $C^{\infty}$ with respect to $t$ and real-analytic with respect to $x$. He also showed the non-existence of sufficiently smooth null-solutions. (As for

Receive April 28, 1995.

The research was supported in part by Grant-in-Aid for Scientific Research (No. 05640168, No. 06640222), Ministry of Education, Science and Culture (Japan). 
distribution null-solutions, see [4]). This class of operators is defined in terms of four conditions. He gave a conjecture that if the third condition is violated, then there exists a $C^{\infty}$ null-solution.

In this article, we construct an asymptotic solution of $P u=0$ in the form

$$
u(t, x):=\exp \left(-\sum_{j=0}^{M} \frac{\lambda[j](x)}{\mu[j]} t^{-\mu[j]}\right) \cdot t^{\lambda[M+1](x)} \cdot \sum_{l=0}^{\infty} t^{l / q} \sum_{p=0}^{l m}(\log t)^{p} v_{l, p}(x),
$$

where

(i) $M$ is a non-negative integer, and $q$ is a positive integer,

(ii) $\mu[j](j=0,1, \ldots, M)$ are positive rational numbers such that $\mu[0]>$ $\mu[1]>\cdots>\mu[M]>0$.

(iii) $\lambda[j](j=0,1, \ldots, M+1)$ and $v_{l, p}(l \geq 0 ; 0 \leq p \leq l m)$ are realanalytic in a fixed open neighborhood of $0 \in \mathbf{R}^{n}$,

for a class of operators wider than that considered in [3].

Further, using these asymptotic solutions, we prove the conjecture in [3] mentioned above under an additional assumption. The $C^{\infty}$ null-solution constructed here is one of the most fastly decaying nontrivial solutions of $P u=0$.

In Section 2, we give the statements of the main theorems. After giving some preliminaries in Section 3, we prove the main theorems in Sections 4 and 5.

Notations:

(i) The set of all integers (resp. nonnegative integers) is denoted by $\mathbf{Z}$ (resp. $\mathbf{N}$ ). Put $\mathbf{N} / q:=\{p / q: p \in \mathbf{N}\}$ for a positive integer $q$, and put $\mathbf{Z} / q$ similarly.

(ii) Put $\vartheta:=t \partial_{t}$.

(iii) For a bounded domain $\Omega$ in $\mathbf{C}^{n}$, we denote by $\mathscr{O}(\Omega)$ the set of all holomorphic functions on $\Omega$.

(iv) The space of the Schwartz distributions on $U$ is denoted by $\mathscr{D}^{\prime}(U)$.

(v) For a complete locally convex topological vector space $E$, put

$$
\begin{aligned}
& C_{f l a t}^{N}([0, T] ; E):=\left\{f \in C^{N}([0, T] ; E)\right. \\
& \left.\qquad\left.\frac{d^{j} f}{d t^{j}}\right|_{t=0}=0 \text { for } 0 \leq j \leq N-1\right\} .
\end{aligned}
$$

(vi) Put $(\lambda)_{j}:=\Pi_{l=0}^{j-1}(\lambda-l)$ for $\lambda \in \mathbf{C}$ and $j \in \mathbf{N}$.

(vii) For a commutative ring $R$, the ring of polynomials of $\lambda$ with the coefficients belonging to $R$ is denoted by $R[\lambda]$. The degree of $F \in R[\lambda]$ is denoted by $\operatorname{deg}_{\lambda} F$. 


\section{§2. Statement of the main result}

Let $q$ be a positive integer, $\Omega$ be a bounded domain in $\boldsymbol{C}^{n}$ that includes the origin 0 , and $T$ be a positive real number. Consider a linear partial differential operator of the form (1.1). We assume only the following weaker condition on the coefficients.

$$
a_{j, \alpha} \in \widehat{\mathscr{F}}_{q}([0, T] ; \mathscr{O}(\Omega))(j+|\alpha| \leq m),
$$

where

$$
\begin{aligned}
\mathscr{F}_{q}([0, T] ; \mathcal{O}(\Omega)):= & \left\{\phi \in C^{\infty}((0, T] ; \mathscr{O}(\Omega))\right. \\
& \left.:\left[s \mapsto \phi\left(s^{q}\right)\right] \in C^{\infty}\left(\left[0, T^{1 / q}\right] ; \mathscr{O}(\Omega)\right)\right\}, \\
\widehat{\mathscr{F}}_{q}([0, T] ; \mathscr{O}(\Omega)):= & \left\{\phi \in C^{\infty}((0, T] ; \mathscr{O}(\Omega))\right. \\
& \left.: t^{M} \phi(t) \in \mathscr{F}_{q}([0, T] ; \mathscr{O}(\Omega)) \text { for some } M \in \mathbf{N}\right\} .
\end{aligned}
$$

Let $r(j, \alpha)$ be the generalized vanishing order of $a_{j, \alpha}$ on the hypersurface $\Sigma:=\{(0, x): x \in \Omega\}$, that is

$$
r(j, \alpha):=\sup \left\{r \in \mathbf{Z} / q: t^{-r} a_{j, \alpha} \in \mathscr{F}_{q}([0, T] ; \mathscr{O}(\Omega))\right\} .
$$

If $r(j, \alpha)=\infty$, then we redefine $r(j, \alpha):=R$ for a sufficiently large $R(R:=$ $\max \{r(j, \alpha): r(j, \alpha)<\infty\}+1$ will suffice). Put

$$
\tilde{a}_{j, \alpha}(t, x):=t^{-r(j, \alpha)} a_{j, \alpha}(t, x) \quad\left(\in \mathscr{F}_{q}([0, T] ; \mathscr{O}(\Omega))\right) .
$$

Note that if $r(j, \alpha)<R$, then $\tilde{a}_{j, \alpha}(0, x) \not \equiv 0$.

Associating a weight $\omega(j, \alpha):=j-r(j, \alpha)$ to each differential monomial $a_{j, \alpha}(t, x) \partial_{t}^{j} \partial_{x}^{\alpha}$, we draw a Newton polygon $\Delta(P)$ using the points $(j+|\alpha|$, $\omega(j, \alpha))(j+|\alpha| \leq m)$ in $(u, v)$-plane as follows.

Definition 2.1 ([3]). (1) Put

$$
\Delta(P):=\operatorname{ch}\left(\bigcup_{j+|\alpha| \leq m}\left\{(u, v) \in \mathbf{R}^{2}: u \leq j+|\alpha|, v \geq-\omega(j, \alpha)\right\}\right),
$$

where $\operatorname{ch}(A)$ denotes the convex hull of $A$. This is called the Newton polygon of $P$. 


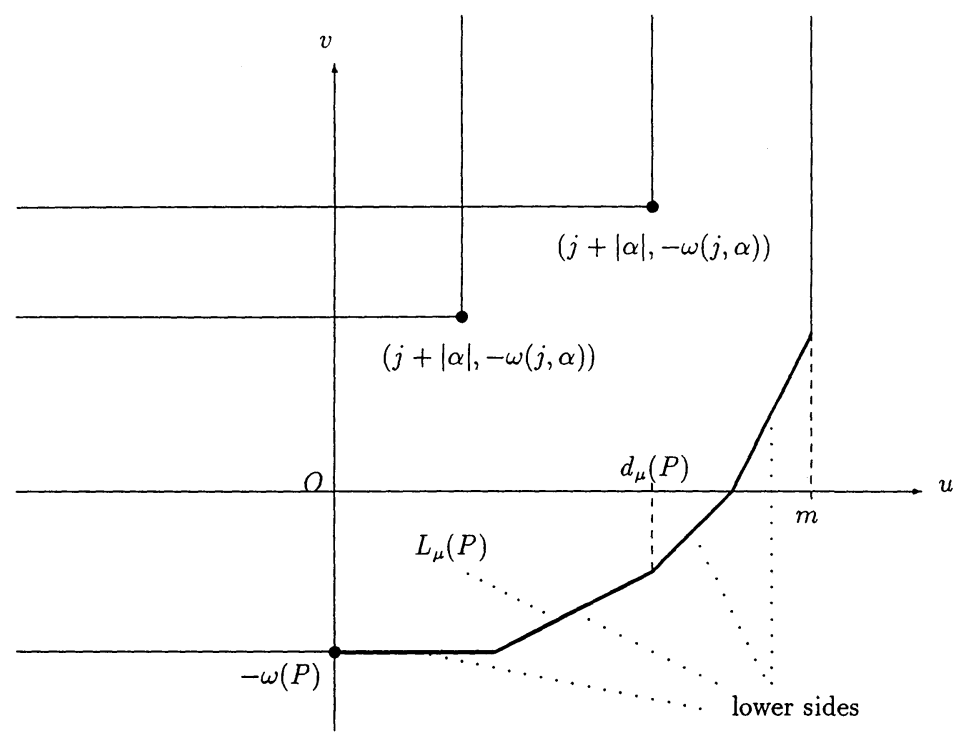

FIgURE 1. Newton polygon of $P: \Delta(P)$

(2) Put

$\hat{V}=\hat{V}(P):=\left\{(j, \alpha) \in \mathbf{N} \times \mathbf{N}^{n}:(j+|\alpha|,-\omega(j, \alpha))\right.$ is a vertex of $\left.\Delta(P)\right\}$.

(3) Put

$$
\omega=\omega(P):=\max \{\omega(j, \alpha) \in \mathbf{R}: j+|\alpha| \leq m\},
$$

which is the maximum weight of $P$.

(4) The boundary of $\Delta(P) \cap([0, \infty) \times \mathbf{R})$ is the union of two vertical half-lines and a finite number of compact line segments with distinct slopes. Each of these compact line segments is called a lower side of $\Delta(P)$. The set of the slopes of the lower sides of $\Delta(P)$ is denoted by $S=S(P)(\subset \mathbf{Q})$. For $\mu \in S(P)$, the lower side of $\Delta(P)$ with slope $\mu$ is denoted by $L_{\mu}=L_{\mu}(P)$. Put

$$
I_{\mu}=I_{\mu}(P):=\left\{(j, \alpha) \in \mathbf{N} \times \mathbf{N}^{n}:(j+|\alpha|,-\omega(j, \alpha)) \in L_{\mu}(P)\right\} .
$$

Let the right end points of $L_{\mu}(P)$ be $\left(u_{1}, v_{1}\right)$. We put $d_{\mu}(P):=u_{1}$, and call it the degree of the slope $\mu$.

If $0 \notin S$, we put $L_{0}(P):=\{(0,-\omega(0,0))\}=\{(0,-\omega(P))\} \subset \mathbf{R}^{2}, I_{0}(P)$ $:=\{(0,0)\} \subset \mathbf{N} \times \mathbf{N}^{n}$, and $d_{0}(P):=0$.

By the use of these notions, Fuchsian operators in the sense of M. S. Baouendi and C. Goulaouic [1] are characterized as follows. (In fact, they assumed that the 
coefficients belong to $C^{m}([0, T] ; \mathscr{O}(\Omega))$. This difference is, however, not essential and hence we ignore the difference of the classes of coefficients.)

Proposition 2.2. The operator $P$ is Fuchsian if and only if $\omega(P) \geq 0, S(P)=$ $\{0\}$, and there exist no $(j, \alpha) \in I_{0}(P)$ such that $\alpha \neq 0$.

We consider a class of operators wider than the class of Fuchsian operators. First, we assume the following condition.

(A-1) For all $\mu \in S(P)$, there exist no $(j, \alpha) \in I_{\mu}(P)$ such that $\alpha \neq 0$.

Definition 2.3. For $\mu \in S(P)$ with $\mu>0$, we put

$$
\mathscr{C}_{\mu}[P](x ; \lambda):=\sum_{(j, 0) \in I_{\mu}(P)} \tilde{a}_{j, 0}(0, x) \lambda^{\prime} \in \mathscr{O}(\Omega)[\lambda]
$$

We also put

$$
\mathscr{C}_{0}[P](x ; \lambda):=\sum_{(j, 0) \in I_{0}(P)} \tilde{a}_{j, 0}(0, x)(\lambda)_{j} \in \mathscr{O}(\Omega)[\lambda]
$$

The polynomial $\mathscr{C}_{\mu}[P]$ of $\lambda$ is called the indicial polynomial of $P$ associated with the slope $\mu \in S(P) \cup\{0\}$. Note that $d_{\mu}(P)=\operatorname{deg}_{\lambda} \mathscr{C}_{\mu}[P]$.

For $\mu \in S(P) \cup\{0\}$, we consider the following condition.

$(\mathrm{A}-2 ; \mu) \quad$ If $(j, 0) \in \hat{V}(P)$ and $j \geq d_{\mu}(P)$, then $\tilde{a}_{j, 0}(0,0) \neq 0$.

This is equivalent to the following.

$($ A-2; $\mu)$ For every $\nu \in S(P)$ with $\nu \geq \mu$, the coefficient of the top order term of $\mathscr{C}_{\nu}[P](x ; \lambda) \in \mathscr{O}(\Omega)[\lambda]$ does not vanish at $x=0$.

Remark 2.4. Note that if $(j, 0) \in \hat{V}(P)$, then $\tilde{a}_{j, 0}(0, x) \not \equiv 0$. Thus, the condition $(\mathrm{A}-2 ; \mu)$ is a kind of non-degeneracy at $x=0$. Further, the condition $(\mathrm{A}-2 ; \mu)$ for $\mu>0$ is weaker than the condition $(A-2 ; 0)$, and $(A-2 ; 0)$ is equivalent to $(\mathrm{A}-2)$ in [3].

Now, the following is one of the three main theorems in this article.

Theorem 2.5. Assume that $P$ satisfies (A-0) and (A-1). Let $\mu_{0} \in S(P) \cap \mathbf{N} / q$, $\mu_{0}>0$, and assume the condition $\left(\mathrm{A}-2 ; \mu_{0}\right)$. If $\lambda_{0}$ is a simple root of $\mathscr{C}_{\mu_{0}}[P](0 ; \lambda)=$ 0 , then there exist

(i) $M \in \mathbf{N}$, 
(ii) $\mu[j] \in \mathbf{N} / q(j=0,1, \ldots, M)$, where $\mu_{0}=\mu[0]>\mu[1]>\cdots>\mu[M]>0$,

(iii) a subdomain $\Omega_{0}$ of $\Omega$ including 0 ,

(iv) $\lambda[j] \in \mathscr{O}\left(\Omega_{0}\right)(j=0,1, \ldots, M+1)$, where $\lambda[0](0)=\lambda_{0}$, such that the following holds.

For an arbitrarily given $v_{0,0}(x) \in \mathscr{O}\left(\Omega_{0}\right)$, there exists $v_{l, p}(x) \in \mathscr{O}\left(\Omega_{0}\right) \quad(l \geq 0 ; 0$ $\leq p \leq l m$ ) such that a formal series

$$
u(t, x):=\exp \left(-\sum_{j=0}^{M} \frac{\lambda[j](x)}{\mu[j]} t^{-\mu[j]}\right) \cdot t^{\lambda[M+1](x)} \cdot \sum_{l=0}^{\infty} t^{l / q} \sum_{p=0}^{l m}(\log t)^{p} v_{l, p}(x)
$$

is an asymptotic solution of $P u=0$. That is, for every $N \in \mathbf{N}$ there holds

$$
\begin{aligned}
t^{-\lambda[M+1](x)} \cdot \exp \left(\sum_{j=0}^{M} \frac{\lambda[j](x)}{\mu[j]} t^{-\mu[j]}\right) \cdot P\left(\exp \left(-\sum_{j=0}^{M} \frac{\lambda[j](x)}{\mu[j]} t^{-\mu[j]}\right)\right. \\
\left.\quad \times t^{\lambda[M+1](x)} \cdot \sum_{l=0}^{N} t^{l / q} \sum_{p=0}^{l m}(\log t)^{p} v_{l, p}(x)\right)=o\left(t^{N / q-r_{0}}\right),
\end{aligned}
$$

with some $r_{0} \in \mathbf{N}$.

This theorem shall be proved in Section 4. We shall also give a proposition which corresponds to the case of $\mu_{0}=0$ and $M=-1$.

Remark 2.6. Even if $\mu_{0} \in S(P)$ but $\mu_{0} \notin \mathbf{N} / q$, we can retake another $q$ such that $\mu_{0} \in \mathbf{N} / q$ and (A-0) is satisfied. Hence, we can always apply this theorem with this new $q$.

Next, we consider the following condition for $\mu \in S(P)$.

$(\mathrm{A}-6 ; \mu)$ If $\nu \in S(P)$ and $\nu>\mu$, then all non-zero roots $\lambda$ of $\mathscr{C}_{\nu}[P](0 ; \lambda)=$ 0 satisfy $\operatorname{Re} \lambda<0$. Further, there exists $\lambda_{0} \in \mathbf{C}$ which satisfies the following.

(i). $\operatorname{Re} \lambda_{0}>0$

(ii) $\lambda_{0}$ is a simple root of $\mathscr{C}_{\mu}[P](0 ; \lambda)=0$ and the other roots $\lambda$ satisfy $\operatorname{Re} \lambda<\operatorname{Re} \lambda_{0}$.

Remark 2.7. In this section, we define only the conditions (A-0), (A-1), $(\mathrm{A}-2 ; \mu)$, and $(\mathrm{A}-6 ; \mu)$. This apparently strange numbering is for the consistency with [3]. We shall introduce another condition (A-3) in Section 5.

Using the theorem above, we can show the existence theorem of smooth null-solutions, which is the second of the main theorems. 
THEOREM 2.8. Assume the conditions (A-0), (A-1), (A-2; $\left.\mu_{0}\right)$, and $\left(\mathrm{A}-6 ; \mu_{0}\right)$ for some $\mu_{0} \in S(P)$ with $\mu_{0}>0$. Then, $P$ has a $C^{\infty}$ null-solution at $(0,0)$.

The $C^{\infty}$ null-solution given in this theorem is one of the most fastly decaying nontrivial solutions as $t \rightarrow+0$. In fact, we have the following theorem, which is the last of the main theorems.

Theorem 2.9. Assume the conditions (A-0), (A-1), (A-2; $\left.\mu_{0}\right)$, and (A-6; $\left.\mu_{0}\right)$ for some $\mu_{0} \in S(P)$ with $\mu_{0}>0$. Assume that $u$ is a $C^{0}$ solution of $P u=0$ for $t>0$. If there exist $\delta>\operatorname{Re} \lambda_{0}$ and $C_{0}>0$ such that the inequality

$$
|u(t, x)| \leq C_{0} \exp \left(-\frac{\delta}{\mu_{0}} t^{-\mu_{0}}\right)
$$

holds for $t>0$ in a neighborhood of $(0,0)$, then $u=0$ for $t>0$ in a neighborhood of $(0,0)$.

Theorems 2.8 and 2.9 shall be proved in Section 5 .

Finally, let us consider a typical example.

EXAmple 2.10. First, we consider the following ordinary differential operator decomposed into first order operators.

$P_{0}:=t^{d}\left(t^{k_{1}} \vartheta-\lambda_{1}(t, x)\right) \cdots\left(t^{k_{r}} \vartheta-\lambda_{r}(t, x)\right)\left(\partial_{t}-\tilde{\lambda}_{r+1}(t, x)\right) \cdots\left(\partial_{t}-\tilde{\lambda}_{m}(t, x)\right)$, where $m, r, d \in \mathbf{N}, 0 \leq r \leq m, k_{j} \in \mathbf{N}(1 \leq j \leq r) \quad$ and $\quad \lambda_{j}, \tilde{\lambda}_{l} \in C^{\infty}([0, T]$; $\mathscr{O}(\Omega))(1 \leq j \leq r ; r+1 \leq l \leq m)$. Assume that $\lambda_{j}(0, x) \not \equiv 0(1 \leq j \leq r)$ and $k_{1} \geq k_{2} \geq \cdots \geq k_{r} \geq 0$. For this operator, $S\left(P_{0}\right)=\left\{k_{1}, \ldots, k_{r}, 0\right\}$ if $r<m$, and $S\left(P_{0}\right)=\left\{k_{1}, \ldots, k_{m}\right\}$ if $r=m$. The condition $(\mathrm{A}-1)$ is trivially satisfied, and the condition $(\mathrm{A}-2 ; \mu)$ is " if $k_{j}>\mu$ then $\lambda_{j}(0,0) \neq 0$ ”. We can also show that

$$
\mathscr{C}_{\mu}\left[P_{0}\right](x ; \lambda)=\prod_{j: k_{j}>\mu}\left(-\lambda_{j}(0, x)\right) \cdot \prod_{\jmath: k_{j}=\mu}\left(\lambda-\lambda_{j}(0, x)\right) \cdot \lambda^{h(\mu)+m-r}
$$

for $\mu \in S\left(P_{0}\right)$ with $\mu>0$, where $h(\mu)$ is the number of $k_{j}$ 's that satisfy $k_{j}<\mu$. Thus, the condition $\left(\mathrm{A}-6 ; \mu_{0}\right)$ for $\mu_{0}>0$ is the following.

If $k_{j}>\mu_{0}$ then $\operatorname{Re} \lambda_{j}(0,0)<0$. Further, there exists $j_{0}$ such that

(i) $k_{j_{0}}=\mu_{0}$,

(ii) $\operatorname{Re} \lambda_{\jmath_{0}}(0,0)>0$,

(iii) If $k_{j}=\mu_{0}$ and $j \neq j_{0}$, then $\operatorname{Re} \lambda_{j}(0,0)<\operatorname{Re} \lambda_{j_{0}}(0,0)$.

Next, we consider a partial differential operator. Put $\mu_{j}:=0(1 \leq j \leq m-$ $r)$ and $\mu_{m-r+j}:=k_{r+1-j}(1 \leq j \leq r)$. Also put $\omega_{j}:=d+\sum_{l=1}^{j} \mu_{l}(0 \leq j \leq m)$. Consider an operator 


$$
P=P_{0}+\sum_{j=0}^{m} t^{\omega_{j}+1} B_{j}\left(t, x ; \vartheta, \partial_{x}\right),
$$

where $B_{j}\left(t, x ; \vartheta, \partial_{x}\right)=\sum_{|\alpha| \leq j} b_{j, \alpha}(t, x) \partial_{x}^{\alpha} \vartheta^{j-|\alpha|}$ and $b_{j, \alpha} \in C^{\infty}([0, T] ; \mathscr{O}(\Omega))$ Then, $P$ satisfies the condition (A-1), and there hold $\Delta(P)=\Delta\left(P_{0}\right), S(P)=$ $S\left(P_{0}\right), \mathscr{C}_{\mu}[P]=\mathscr{C}_{\mu}\left[P_{0}\right]$. (See Lemma 3.1.) Hence, $P$ satisfies the condition (A-2; $\left.\mu_{0}\right)$ (resp. (A-6; $\left.\left.\mu_{0}\right)\right)$, if and only if $P_{0}$ satisfies $\left(\mathrm{A}-2 ; \mu_{0}\right)\left(\right.$ resp. $\left(\mathrm{A}-6 ; \mu_{0}\right)$ ).

\section{§3. Preliminaries}

In this section, we give some preliminaries for the proofs of the main theorems.

Let $P$ be an operator (1.1) satisfying (A-0). By $t^{j} \partial_{t}^{j}=\vartheta(\vartheta-1) \ldots(\vartheta-j+1)$ $=(\vartheta)_{j}$, we can easily show the following lemma, which is useful in our arguments.

Lemma 3.1. We can rewrite $P$ as

$$
P=\sum_{j+|\alpha| \leq m} b_{j, \alpha}(t, x) \vartheta^{j} \partial_{x}^{\alpha},
$$

with $b_{j, \alpha} \in \widehat{\mathscr{F}}_{q}([0, T] ; \mathcal{O}(\Omega))$. For this $b_{j, \alpha}$, we define the generalized vanishing order

$$
r^{\prime}(j, \alpha):=\sup \left\{r \in \mathbf{Z} / q: t^{-r} b_{j, \alpha} \in \mathscr{F}_{q}([0, T] ; \mathscr{O}(\Omega))\right\} .
$$

For $\mu \geq 0$, we put $\omega_{\mu}(P):=\max \left\{-r^{\prime}(j, \alpha)+\mu(j+|\alpha|): j+|\alpha| \leq m\right\}$. Then, we have

$$
\begin{aligned}
& \Delta(P)=\operatorname{ch}\left(\bigcup_{j+|\alpha| \leq m}\left\{(u, v) \in \mathbf{R}^{2}: u \leq j+|\alpha|, v \geq r^{\prime}(j, \alpha)\right\}\right), \\
& \hat{V}(P)=\left\{(j, \alpha) \in \mathbf{N} \times \mathbf{N}^{n}:\left(j+|\alpha|, r^{\prime}(j, \alpha)\right) \text { is a vertex of } \Delta(P)\right\}, \\
& \omega(P)=\max \left\{-r^{\prime}(j, \alpha) \in \mathbf{R}: j+|\alpha| \leq m\right\}=\omega_{0}(P), \\
& I_{\mu}(P)=\left\{(j, \alpha) \in \mathbf{N} \times \mathbf{N}^{n}:-r^{\prime}(j, \alpha)+\mu(j+|\alpha|)=\omega_{\mu}(P)\right\} .
\end{aligned}
$$

Further, the condition (A-1) is stated as follows:

(A-1) For every $\mu \in S(P)$, if $-r^{\prime}(j, \alpha)+\mu(j+|\alpha|)=\omega_{\mu}(P)$, then $\alpha=0$.

Under (A-1), there holds

$$
\begin{aligned}
\mathscr{C}_{\mu}[P](x ; \lambda) & =\left.\sum_{j=0}^{m}\left\{b_{j, 0}(t, x) t^{\omega_{\mu}(P)-\mu j}\right\}\right|_{t=0} \lambda^{j} \\
& = \begin{cases}{\left.\left[t^{\omega_{\mu}(P)} e^{\lambda t^{-\mu} / \mu} P\left(e^{-\lambda t^{-\mu} / \mu}\right)\right]\right|_{t=0}} & (\mu>0), \\
{\left.\left[t^{\omega(P)} t^{-\lambda} P\left(t^{\lambda}\right)\right]\right|_{t=0}} & (\mu=0),\end{cases}
\end{aligned}
$$


and the condition $(\mathrm{A}-2 ; \mu)$ is stated as follows:

$(\mathrm{A}-2 ; \mu)$ If $(j, 0) \in \hat{V}(P)$ and $j \geq d_{\mu}(P)$, then $\left.\left\{b_{j, 0}(t, 0) t^{-r^{\prime}(j, 0)}\right\}\right|_{t=0} \neq 0$.

It is convenient to consider the operator in the form (3.1) rather than the form (1.1).

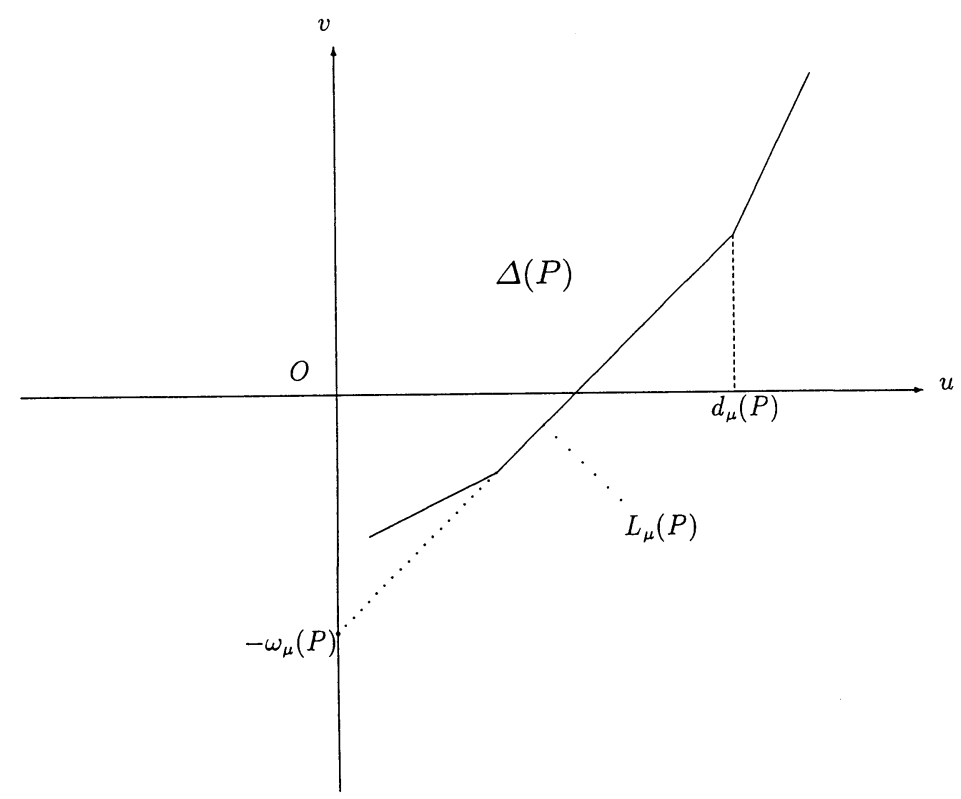

Figure 2. $\omega_{\mu}(P)$

Remark 3.2. For $\mu \geq 0$, we can define $\mathscr{C}_{\mu}[P]$ by (3.2), even if $\mu \notin S$. If $\mu \in S$ and $\mu>0$, then $\mathscr{C}_{\mu}[P]$ has more than one term as a polynomial of $\lambda$. If $\mu \notin$ $S$ and $\mu>0$, then $\mathscr{C}_{\mu}[P]$ has only one term.

The key tool for the proofs of main thorems is the following type of transformation of operators.

Lemma 3.3. Assume that an operator $P$ of the form (1.1) (or (3.1)) satisfies the conditions (A-0) and (A-1). Let $\mu \in S(P) \cap \mathbf{N} / q, \mu>0$, and assume (A-2; $\mu$ ). Let $\lambda_{1}$ be a simple root of $\mathscr{C}_{\mu}[P](0 ; \lambda)=0$. Take a subdomain $\Omega^{\prime}$ of $\Omega$ including 0 and $\lambda(x) \in \mathscr{O}\left(\Omega^{\prime}\right)$ so that they satisfy $\lambda(0)=\lambda_{1}$ and $\mathscr{C}_{\mu}[P](x ; \lambda(x)) \equiv 0$ on $\Omega^{\prime}$. If we put 


$$
P^{\prime}:=\exp \left(\frac{\lambda(x)}{\mu} t^{-\mu}\right) \circ P \circ \exp \left(-\frac{\lambda(x)}{\mu} t^{-\mu}\right)
$$

then $P^{\prime}$ is an operator on $[0, T] \times \Omega^{\prime}$ of the form (1.1) and satisfies the following:

(a) The operator $P^{\prime}$ satisfies (A-0) and (A-1).

(b) $S\left(P^{\prime}\right) \cap(\mu, \infty)=S(P) \cap(\mu, \infty)$.

(c) $\mathscr{C}_{\nu}\left[P^{\prime}\right](x ; \cdot)=\mathscr{C}_{\nu}[P](x ; \cdot)$ for every $\nu>\mu$ and $x \in \Omega^{\prime}$.

(d) There holds $\mathscr{C}_{\mu}\left[P^{\prime}\right](x ; \lambda)=\mathscr{C}_{\mu}[P](x ; \lambda+\lambda(x))$. Further, if $d_{\mu}(P)>1$, then $\mu \in S\left(P^{\prime}\right)$; if $d_{\mu}(P)=1$, then $\mu \notin S\left(P^{\prime}\right)$.

(e) There exists $\mu^{\prime}<\mu$ such that $\mu^{\prime} \in \mathbf{N} / q$ and $S\left(P^{\prime}\right) \cap[0, \mu)=\left\{\mu^{\prime}\right\}$.

(f) $d_{\mu^{\prime}}\left(P^{\prime}\right)=1$ and $P^{\prime}$ satisfies $\left(\mathrm{A}-2 ; \mu^{\prime}\right)$.

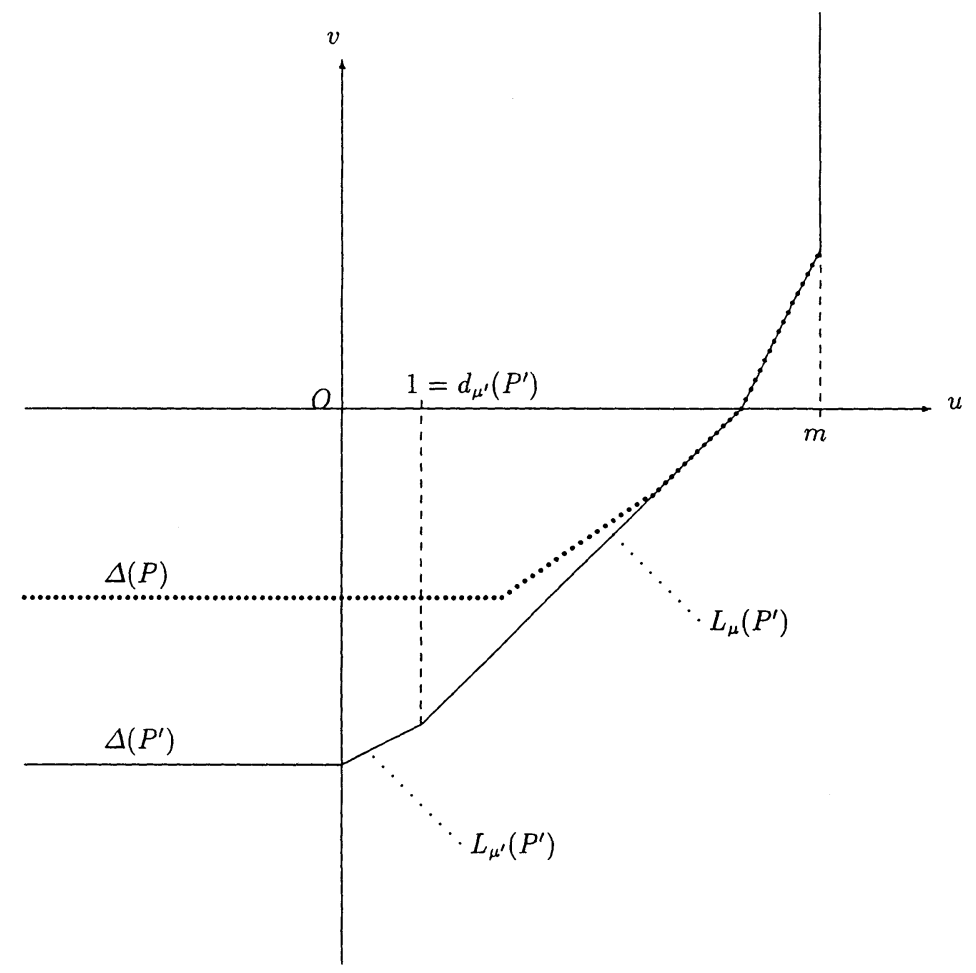

The upper part of the dotted line is $\Delta(P)$.

The upper part of the real line is $\Delta\left(P^{\prime}\right)$.

FIGURE 3. $\Delta\left(P^{\prime}\right)$ and $\Delta(P)$

Proof. First, note that 


$$
\begin{gathered}
\exp \left(\frac{\lambda(x)}{\mu} t^{-\mu}\right) \circ \vartheta \circ \exp \left(-\frac{\lambda(x)}{\mu} t^{-\mu}\right)=\vartheta+\lambda(x) t^{-\mu} \\
\exp \left(\frac{\lambda(x)}{\mu} t^{-\mu}\right) \circ \partial_{x} \circ \exp \left(-\frac{\lambda(x)}{\mu} t^{-\mu}\right)=\partial_{x}+\frac{-\lambda_{x}(x)}{\mu} t^{-\mu} .
\end{gathered}
$$

From these, it is easy to see that $P^{\prime}$ is an operator of the form (3.1) and satisfies the conditions $(\mathrm{A}-0),(\mathrm{A}-1)$, and $(\mathrm{A}-2 ; \mu)$. It is also easy to see that there hold the conclusions (b), (c). Further, we have $\mathscr{C}_{\mu}\left[P^{\prime}\right](x ; \lambda)=\mathscr{C}_{\mu}[P](x ; \lambda+\lambda(x))$. Since $\mathscr{C}_{\mu}\left[P^{\prime}\right](x ; 0) \equiv 0$ and since $\left(\partial_{\lambda} \mathscr{C}_{\mu}\left[P^{\prime}\right]\right)(0 ; 0)=\left(\partial_{\lambda} \mathscr{C}_{\mu}[P]\right)\left(0 ; \lambda_{1}\right) \neq 0$, we have $(1,0, \ldots, 0) \in \hat{V}\left(P^{\prime}\right)\left(\subset \mathbf{N} \times \mathbf{N}^{n}\right)$. Hence, if $d_{\mu}(P)>1$, then $\mu \in S\left(P^{\prime}\right)$; if $d_{\mu}(P)=1$, then $\mu \notin S\left(P^{\prime}\right)$. Further, there exists $\mu^{\prime} \in \mathbf{N} / q$ such that $\mu^{\prime}<\mu$, $S\left(P^{\prime}\right) \cap[0, \mu)=\left\{\mu^{\prime}\right\}$, and $d_{\mu^{\prime}}\left(P^{\prime}\right)=1$. The condition $(\mathrm{A}-2 ; \mu)$ and the fact that $\left(\partial_{\lambda} \mathscr{C}_{\mu}\left[P^{\prime}\right]\right)(0 ; 0) \neq 0$ imply $\left(\mathrm{A}-2 ; \mu^{\prime}\right)$.

By an iterative use of this lemma, we have the following.

Proposition 3.4. Assume that $P$ satisfies (A-0) and (A-1). Let $\mu_{0} \in S(P) \cap$ $\mathbf{N} / q, \mu_{0}>0$, and assume $\left(\mathrm{A}-2 ; \mu_{0}\right)$. Let $\lambda_{0}$ be a simple root of $\mathscr{C}_{\mu_{0}}[P](0 ; \lambda)=0$. Then, there exist

(i) $M \in \mathbf{N}$,

(ii) $\mu[j] \in \mathbf{N} / q(j=0,1, \ldots, M)$, where $\mu_{0}=\mu[0]>\mu[1]>\cdots>\mu[M]>0$,

(iii) a subdomain $\Omega_{M+1}$ of $\Omega$ including 0 ,

(iv) $\lambda[j] \in \mathscr{O}\left(\Omega_{M+1}\right)(j=0,1, \ldots, M)$, where $\lambda[0](0)=\lambda_{0}$,

such that the operator

$$
P^{(M+1)}:=\exp \left(\sum_{j=0}^{M} \frac{\lambda[j](x)}{\mu[j]} t^{-\mu[j]}\right) \circ P \circ \exp \left(-\sum_{j=0}^{M} \frac{\lambda[j](x)}{\mu[j]} t^{-\mu[j]}\right)
$$

is an operator on $[0, T] \times \Omega_{M+1}$ of the form (1.1) and satisfies the following:

(a) The operator $P^{(M+1)}$ satisfies (A-0) and (A-1).

(b) $S\left(P^{(M+1)}\right) \cap\left(\mu_{0}, \infty\right)=S(P) \cap\left(\mu_{0}, \infty\right)$.

(c) $\mathscr{C}_{\nu}\left[P^{(M+1)}\right](x ; \cdot)=\mathscr{C}_{\nu}[P](x ; \cdot)$ for every $\nu>\mu_{0}$ and $x \in \Omega_{M+1}$.

(d) There holds $\mathscr{C}_{\mu_{0}}\left[P^{(M+1)}\right](x ; \lambda)=\mathscr{C}_{\mu_{0}}[P](x ; \lambda+\lambda[0](x))$. If $d_{\mu_{0}}(P)>1$, then $\mu_{0} \in S\left(P^{(M+1)}\right)$; if $d_{\mu_{0}}(P)=1$, then $\mu_{0} \notin S\left(P^{(M+1)}\right)$.

(e) $S\left(P^{(M+1)}\right) \cap\left[0, \mu_{0}\right)=\{0\}$.

(f) $d_{0}\left(P^{(M+1)}\right)=1$ and $P^{(M+1)}$ satisfies $(\mathrm{A}-2 ; 0)$. 


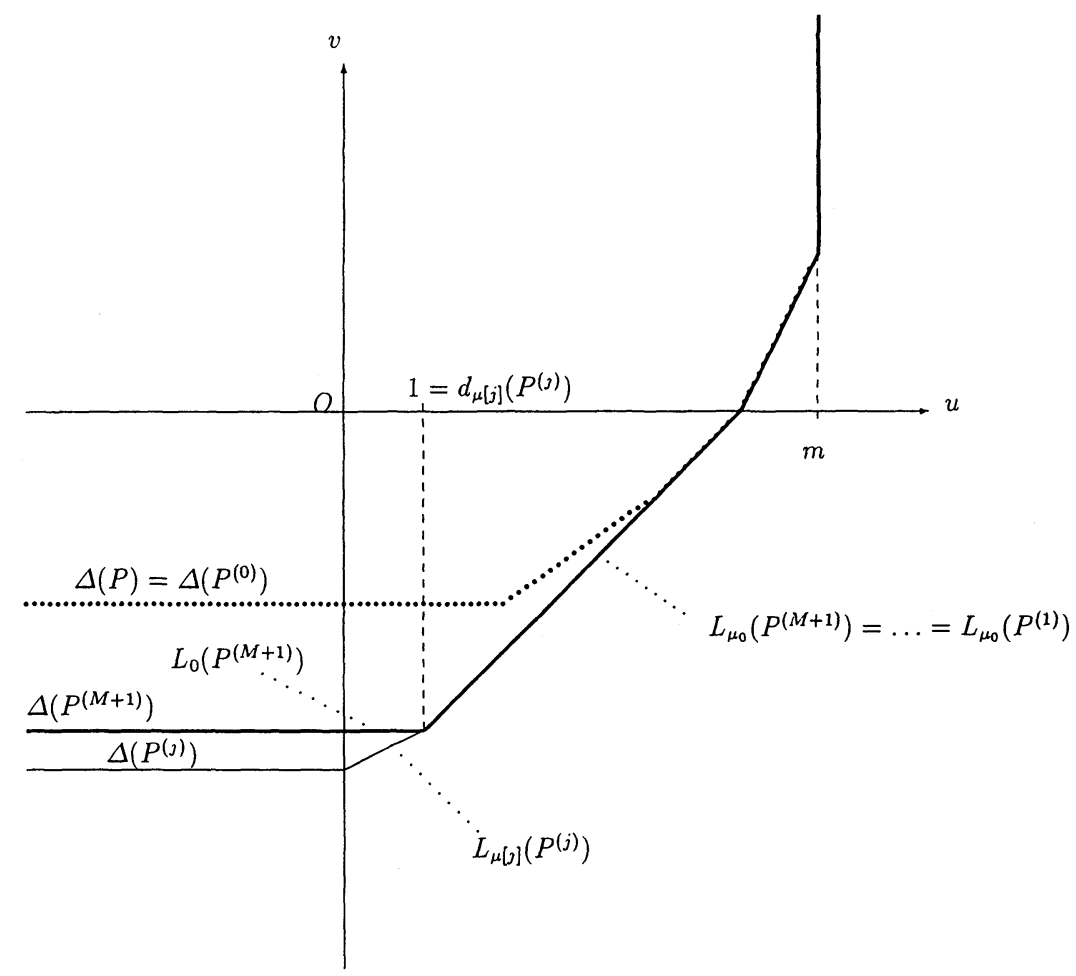

The upper part of the dotted line is $\Delta(P)=\Delta\left(P^{(0)}\right)$.

The upper part of the real line is $\Delta\left(P^{(j)}\right)(1 \leq j \leq M)$.

The upper part of the bold real line is $\Delta\left(P^{(M+1)}\right)$.

Figure 4. $\Delta(P)=\Delta\left(P^{(0)}\right)$ and $\Delta\left(P^{(M+1)}\right) \subset \cdots \subset \Delta\left(P^{(1)}\right)$

Proof. Since $\lambda_{0}$ is a simple root, we can take a subdomain $\Omega_{1}$ of $\Omega$ including 0 and $\lambda[0](x) \in \mathscr{O}\left(\Omega_{1}\right)$ such that they satisfy $\lambda[0](0)=\lambda_{0}$ and $\mathscr{C}_{\mu_{0}}[P](x$; $\lambda[0](x)) \equiv 0$ on $\Omega_{1}$.

Put $P^{(0)}:=P$ and $\mu[0]:=\mu_{0}$. If we put

$$
P^{(1)}:=\exp \left(\frac{\lambda[0](x)}{\mu[0]} t^{-\mu[0]}\right) \circ P^{(0)} \circ \exp \left(-\frac{\lambda[0](x)}{\mu[0]} t^{-\mu[0]}\right)
$$

then by Lemma 3.3 , the operator $P^{(1)}$ is also an operator of the form $(1.1)$ on $[0, T]$ $\times \Omega_{1}$ and satisfies the following:

(a) The operator $P^{(1)}$ satisfies $(\mathrm{A}-0)$ and $(\mathrm{A}-1)$. 
(b) $S\left(P^{(1)}\right) \cap(\mu[0], \infty)=S\left(P^{(0)}\right) \cap(\mu[0], \infty)$.

(c) $\mathscr{C}_{\nu}\left[P^{(1)}\right](x ; \cdot)=\mathscr{C}_{\nu}\left[P^{(0)}\right](x ; \cdot)$ for every $\nu>\mu[0]$ and $x \in \Omega_{1}$.

(d) There holds $\mathscr{C}_{\mu[0]}\left[P^{(1)}\right](x ; \lambda)=\mathscr{C}_{\mu[0]}\left[P^{(0)}\right](x ; \lambda+\lambda[0](x))$. If $d_{\mu[0]}\left(P^{(0)}\right)$ $>1$, then $\mu[0] \in S\left(P^{(1)}\right)$; if $d_{\mu[0]}\left(P^{(0)}\right)=1$, then $\mu[0] \notin S\left(P^{(1)}\right)$.

(e) There exists $\mu[1]<\mu[0]$ such that $\mu[1] \in \mathbf{N} / q$ and $S\left(P^{(1)}\right) \cap[0$, $\mu[0])=\{\mu[1]\}$.

(f) $d_{\mu[1]}\left(P^{(1)}\right)=1$ and $P^{(1)}$ satisfies $(\mathrm{A}-2 ; \mu[1])$.

By (f), we have $\mathscr{C}_{\mu[1]}\left[P^{(1)}\right](x ; \lambda)=a[1](x) \lambda-b[1](x)$ for some $a[1], b[1] \in$ $\mathscr{O}\left(\Omega_{1}\right)$ with $a[1](0) \neq 0$.

If $\mu[1]=0$, then put $M=0$. Consider the case when $\mu[1]>0$. We can take a subdomain $\Omega_{2}$ of $\Omega_{1}$ including 0 such that $a[1](x) \neq 0$ on $\Omega_{2}$, and hence we can take $\lambda[1] \in \mathscr{O}\left(\Omega_{2}\right)$ such that $\mathscr{C}_{\mu[1]}\left[P^{(1)}\right](x ; \lambda[1](x)) \equiv 0$ on $\Omega_{2}$.

If we put

$$
P^{(2)}:=\exp \left(\frac{\lambda[1](x)}{\mu[1]} t^{-\mu[1]}\right) \circ P^{(1)} \circ \exp \left(-\frac{\lambda[1](x)}{\mu[1]} t^{-\mu[1]}\right),
$$

then by Lemma 3.3 and by $d_{\mu[1]}\left(P^{(1)}\right)=1$, the operator $P^{(2)}$ is also an operator of the form (1.1) and satisfies the following:

(a) The operator $P^{(2)}$ satisfies $(\mathrm{A}-0)$ and $(\mathrm{A}-1)$.

(b) $S\left(P^{(2)}\right) \cap(\mu[1], \infty)=S\left(P^{(1)}\right) \cap(\mu[1], \infty)$.

(c) $\mathscr{C}_{\nu}\left[P^{(2)}\right](x ; \cdot)=\mathscr{C}_{\nu}\left[P^{(1)}\right](x ; \cdot)$ for every $\nu>\mu[1]$ and $x \in \Omega_{2}$.

(d) There holds $\mathscr{C}_{\mu[1]}\left[P^{(2)}\right](x ; \lambda)=\mathscr{C}_{\mu[1]}\left[P^{(1)}\right](x ; \lambda+\lambda[1](x))=a[1](x) \lambda$, and $\mu[1] \notin S\left(P^{(2)}\right)$.

(e) There exists $\mu[2]<\mu[1]$ such that $\mu[2] \in \mathbf{N} / q$ and $S\left(P^{(2)}\right) \cap[0$, $\mu[1]]=\{\mu[2]\}$.

(f) $d_{\mu[2]}\left(P^{(2)}\right)=1$ and $P^{(2)}$ satisfies $(\mathrm{A}-2 ; \mu[2])$.

We can continue this procedure unless $\mu[j]=0$. Since $\mu[j] \in \mathbf{N} / q$ and $\mu[0]>\mu[1]>\cdots \geq 0$, we necessarily reach $\mu[M+1]=0$.

The following lemma is used to construct each term of infinite series in asymptotic solutions.

Lemma 3.5. Let $Q(x ; \lambda) \in \mathscr{O}(\Omega)[\lambda]$ and $\Lambda \in \mathscr{O}(\Omega)$. Assume that $Q(x ;$ $\Lambda(x)) \neq 0$ on $\Omega$. Then, we can solve the equation

$$
Q(x ; \vartheta) v=t^{\Lambda(x)} \sum_{p=0}^{L} g_{p}(x)(\log t)^{p}, \quad g_{p} \in \mathscr{O}(\Omega)(0 \leq p \leq L)
$$


as $v=t^{\Lambda(x)} \sum_{p=0}^{L} v_{p}(x)(\log t)^{p}, \quad v_{p} \in \mathscr{O}(\Omega)(0 \leq p \leq L)$.

Proof. By an easy calculation, we have

$$
Q(x ; \vartheta)\left(t^{\Lambda(x)}(\log t)^{p}\right)=\sum_{j=0}^{p}\left(\begin{array}{l}
p \\
j
\end{array}\right)\left(\partial_{\lambda}^{j} Q\right)(x ; \Lambda(x)) \cdot t^{\Lambda(x)}(\log t)^{p-j} .
$$

Hence, (3.4) is equivalent to

$$
\begin{aligned}
& Q(x ; \Lambda(x)) \cdot v_{p}(x) \\
& \quad+\sum_{j=1}^{L-p}\left(\begin{array}{c}
p+j \\
j
\end{array}\right)\left(\partial_{\lambda}^{j} Q\right)(x ; \Lambda(x)) \cdot v_{p+j}(x)=g_{p}(x) \quad(p=0,1, \ldots, L) .
\end{aligned}
$$

Thus, by $Q(x ; \Lambda(x)) \neq 0$, we can uniquely determine $v_{L}, v_{L-1}, \ldots, v_{0}$.

\section{§4. Proof of Theorem 2.5}

In this section, we prove Theorem 2.5. First, we give the existence of an asymptotic solution with no exponential factor, which corresponds to the case $\mu_{0}=0$ and $M=-1$ in Theorem 2.5. Although we use only the case when $\operatorname{deg}_{\lambda}$ $\mathscr{C}_{0}[P]=1$ in the proof of main theorems, this proposition has its own value.

Proposition 4.1. Assume that $P$ satisfies (A-0), (A-1), and (A-2;0). Let $\lambda(x) \in \mathscr{O}\left(\Omega_{0}\right)$ satisfy

(i) $\mathscr{C}_{0}[P](x ; \lambda(x)) \equiv 0$ on $\Omega_{0}$,

(ii) $\mathscr{C}_{0}[P](x ; \lambda(x)+l / q) \neq 0$ on $\Omega_{0}$ for $l \in \mathbf{N} \backslash\{0\}$,

for some subdomain $\Omega_{0}$ of $\Omega$ including 0 . Then, for an arbitrarily given $v_{0,0}(x) \in$ $\mathscr{O}\left(\Omega_{0}\right)$, there exist $v_{l, p}(x) \in \mathscr{O}\left(\Omega_{0}\right)(l \geq 0 ; 0 \leq p \leq l m)$ such that

$$
u(t, x):=t^{\lambda(x)} \cdot \sum_{l=0}^{\infty} t^{l / q} \sum_{p=0}^{l m}(\log t)^{p} v_{l, p}(x)
$$

is an asymptotic solution of $P u=0$. That is

$$
t^{-\lambda(x)} P\left(t^{\lambda(x)} \cdot \sum_{l=0}^{N} t^{l / q} \sum_{p=0}^{l m}(\log t)^{p} v_{l, p}(x)\right)=o\left(t^{N / q-\omega(P)}\right),
$$

for every $N \in \mathbf{N}$.

Proof. We can formally expand $P$ with respect to $t$ as 


$$
P=t^{-\omega}\left(\mathscr{C}_{0}[P](x ; \vartheta)+\sum_{h=1}^{\infty} B_{h}\left(x, \partial_{x} ; \vartheta\right) t^{h / q}\right)
$$

where $B_{h}\left(x, \partial_{x} ; \vartheta\right)=\sum_{j+|\alpha| \leq m} b_{h, j, \alpha}(x) \partial_{x}^{\alpha} \vartheta^{j}$ with $b_{h, j, \alpha} \in \mathscr{O}(\Omega)$ and $\omega:=\omega(P)$. Hence, we have only to find $v_{l, p}$ that satisfy

$$
\begin{aligned}
\mathscr{C}_{0}[P] & (x ; \vartheta)\left(t^{\lambda(x)+l / q} \sum_{p=0}^{l m}(\log t)^{p} v_{l, p}(x)\right) \\
& =-\sum_{h=0}^{l-1} B_{l-h}\left(x, \partial_{x} ; \vartheta\right)\left(t^{\lambda(x)+l / q} \sum_{p=0}^{h m}(\log t)^{p} v_{h, p}(x)\right)(l \in \mathbf{N}) .
\end{aligned}
$$

Since

$$
\begin{gathered}
\mathscr{C}_{0}[P](x ; \vartheta)\left(t^{\lambda(x)} v_{0,0}(x)\right)=\mathscr{C}_{0}[P](x ; \lambda(x)) \cdot t^{\lambda(x)} v_{0,0}(x) \equiv 0, \\
\partial_{x}\left(t^{\lambda(x)+l / q}(\log t)^{p} v(x)\right) \\
=t^{\lambda(x)+l / q}(\log t)^{p}\left(\partial_{x} v\right)(x)+t^{\lambda(x)+l / q}(\log t)^{p+1}\left(\partial_{x} \lambda\right)(x) v(x),
\end{gathered}
$$

and since

$$
\mathscr{C}_{0}[P](x ; \lambda(x)+l / q) \neq 0(l \geq 1) \text { on } \Omega_{0},
$$

we can get $v_{l, p}$ with an arbitrarily given $v_{0,0}$ by applying Lemma 3.5 .

Proof of Theorem 2.5. We can apply Proposition 3.4 to $P$. By (f) of the proposition, we have

$$
\mathscr{C}_{0}\left[P^{(M+1)}\right](x ; \lambda)=a[M+1](x) \lambda-b[M+1](x)
$$

for some $a[M+1], b[M+1] \in \mathscr{O}\left(\Omega_{M+1}\right)$ with $a[M+1](0) \neq 0$. Hence, we can take a subdomain $\Omega_{0}$ of $\Omega_{M+1}$ including 0 such that $a[M+1](x) \neq 0$ on $\Omega_{0}$. We can take $\lambda[M+1] \in \mathscr{O}\left(\Omega_{0}\right)$ such that $\mathscr{C}_{0}\left[P^{(M+1)}\right](x ; \lambda[M+1](x)) \equiv 0$ and $\mathscr{C}_{0}\left[P^{(M+1)}\right](x ; \lambda[M+1](x)+l / q) \neq 0$ on $\Omega_{0}$ for $l \in \mathbf{N} \backslash\{0\}$.

By applying Proposition 4.1 to $P^{(M+1)}$, we can construct an asymptotic solution

$$
v=t^{\lambda[M+1](x)} \cdot \sum_{l=0}^{\infty} t^{l / q} \sum_{p=0}^{l m}(\log t)^{p} v_{l, p}(x)
$$

of $P^{(M+1)} v=0$ for an arbitrarily given $v_{0,0} \in \mathscr{O}\left(\Omega_{0}\right)$.

Thus, the proof of Theorem 2.5 is completed. 


\section{\$5. Proof of Theorems 2.8 and 2.9}

In this section, we prove Theorems 2.8 and 2.9 .

First, we introduce another condition (A-3).

(A-3) If $\mu \in S(P)$ and $\mu>0$, then all the non-zero roots $\lambda$ of $\mathscr{C}_{\mu}[P](0$; $\lambda)=0$ satisfy $\operatorname{Re} \lambda<0$.

From the results in [3], we easily get the following theorem, which shall be used later.

Theorem 5.1. Assume the conditions (A-0), (A-1), (A-2;0), and (A-3). Then, there exist $N_{0} \in \mathbf{N}, T_{0}>0$, and a domain $\Omega_{0}$ including 0 for which the following holds:

(1) For every $N \geq N_{0}$ and every $f \in C_{\text {flat }}^{N-\omega(P)}([0, T] ; \mathscr{O}(\Omega))$, there exists a unique $u \in C_{\text {flat }}^{N}\left(\left[0, T_{0}\right] ; \mathscr{O}\left(\Omega_{0}\right)\right)$ such that $P u=f$ on $\left[0, T_{0}\right] \times \Omega_{0}$.

(2) If $u \in t^{N_{0}} \times C^{0}\left([0, T] ; \mathscr{D}^{\prime}\left(\Omega \cap \mathbf{R}^{n}\right)\right)$ and $P u=0$ for $t>0$ in a neighborhood of $(0,0)$, then $u=0$ for $t>0$ in a neighborhood of $(0,0)$. Especially, there exists no sufficiently smooth null-solution for $P$ at $(0,0)$.

In (2) of this theorem, the domain where $u=0$ may depend not only on the domain where $P u=0$ but also on $u$ itself. As for solutions in $C^{0}\left([0, T] ; C^{0}(\Omega\right.$ $\cap \mathbf{R}^{n}$ )), however, we can show the existence of a common domain of uniqueness, by a standard argument as follows.

CoRollary 5.2. Assume the same assumptions as in the theorem above. Then there exists $N_{0} \in \mathbf{N}$ such that for every $T^{\prime} \in(0, T)$ and every open neighborhood $U^{\prime}$ of $0 \in \mathbf{R}^{n}$, there exist $T^{\prime \prime} \in\left(0, T^{\prime}\right)$ and an open neighborhood $U^{\prime \prime}$ of 0 for which the following holds. If $u \in t^{N_{0}} \times C^{0}\left([0, T] ; C^{0}\left(\Omega \cap \mathbf{R}^{n}\right)\right)$ and $P u=0$ on $\left(0, T^{\prime}\right) \times$ $U^{\prime}$, then $u=0$ on $\left(0, T^{\prime \prime}\right) \times U^{\prime \prime}$.

Proof. Put $K:=\left\{u \in t^{N_{0}} \times C^{0}\left([0, T] ; C^{0}\left(\Omega \cap \mathbf{R}^{n}\right)\right): P u=0\right.$ on $\left(0, T^{\prime}\right)$ $\left.\times U^{\prime}\right\}$. This is a closed subspace of a Fréchet space $t^{N_{0}} \times C^{0}\left([0, T] ; C^{0}(\Omega \cap\right.$ $\left.\mathbf{R}^{n}\right)$ ), and hence it is also a Fréchet space. Let $\left\{T_{n}\right\}_{n \in N}$ be a decreasing sequence of positive real numbers converging to 0 and let $\left\{U_{n}\right\}_{n \in N}$ be a fundamental system of open neighborhoods of 0 . Put $L_{n}:=\left\{u \in K: u=0\right.$ on $\left.\left(0, T_{n}\right) \times U_{n}\right\}$, which are closed subspaces of $K$. By Theorem 5.1-(2), there holds $K=\cup_{n=0}^{\infty} L_{n}$. Since a Fréchet space is a Baire space, there exists an $n$ such that $L_{n}$ has an inner point, that is $L_{n}=K$. 
Now, we give a proof of Theorem 2.8 .

Proof of Theorem 2.8. We may assume that $\mu_{0} \in \mathbf{N} / q$ without loss of generality, and we can apply Proposition 3.4 to $P$. The operator $P^{(M+1)}$ satisfies (A-0), $(\mathrm{A}-1)$ and $(\mathrm{A}-2 ; 0)$. By the assumption $\left(\mathrm{A}-6 ; \mu_{0}\right)$ for $P$ and by the conditions (c), (d), (e) in Proposition 3.4, the operator $P^{(M+1)}$ satisfies (A-3). Further, as we have shown in the proof of Theorem 2.5, the operator $P^{(M+1)}$ has a formal solution (4.2) with $v_{0,0} \equiv 1$

If we put

$$
v_{N}:=t^{\lambda[M+1](x)} \cdot \sum_{l=0}^{q N} t^{l / q} \sum_{p=0}^{l m}(\log t)^{p} v_{l, p}(x)
$$

and $g_{N}:=P^{(M+1)}\left(v_{N}\right)$ for sufficiently large $N \in \mathbf{N}$, then we have

$$
g_{N} \in C_{\text {flat }}^{N-r_{0}}\left([0, T] ; \mathscr{O}\left(\Omega_{0}\right)\right),
$$

where $\Omega_{0}$ is a subdomain of $\Omega$ including 0 and $r_{0} \in \mathbf{N}$, both independent of $N$. By Theorem 5.1, we get $w_{N} \in C_{\text {flat }}^{N+\omega\left(P^{(M+1)}\right)-r_{0}}\left(\left[0, T_{0}\right] ; \mathcal{O}\left(\Omega_{0}^{\prime}\right)\right)$ such that $P^{(M+1)}\left(w_{N}\right)=$ $-g_{N}$, where $T_{0}>0$ and $\Omega_{0}^{\prime}$ is a subdomain of $\Omega_{0}$ including 0 . Thus, $v:=v_{N}+$ $w_{N}$ satisfies $P^{(M+1)}(v)=0$ and $t^{-\lambda[M+1](x)} v(t, x) \rightarrow 1(t \rightarrow+0)$. Note that Corol lary 5.2 implies that $v$ is independent of $N$ for sufficiently large $N$ in a neighborhood of $(0,0)$.

Since $\operatorname{Re} \lambda[0](0)>0$ by the assumption, we can easily show that

$$
u(t, x):=\exp \left(-\sum_{j=0}^{M} \frac{\lambda[j](x)}{\mu[j]} t^{-\mu[j]}\right) \cdot v(t, x)
$$

belongs to $C_{\text {flat }}^{\infty}\left(\left[0, T_{0}\right] ; \mathscr{O}\left(\Omega_{0}^{\prime}\right)\right)$. Thus, $u$ is a $C^{\infty}$ null-solution for $P$.

Next, we give a proof of Theorem 2.9.

Proof of Theorem 2.9. If we take $\delta^{\prime}$ as $\delta>\delta^{\prime}>\operatorname{Re} \lambda_{0}$, and if we put $v:=$ $\exp \left(\delta^{\prime} t^{-\mu_{0}} / \mu_{0}\right) u$, then we have $v \in t^{N} \times C^{0}\left([0, T] ; \mathscr{D}^{\prime}\left(\Omega_{0} \cap \mathbf{R}^{n}\right)\right)$ for every $N \in \mathbf{N}$ with some domain $\Omega_{0}$ and $T>0$. We also have

$$
0=P\left(\exp \left(-\frac{\delta^{\prime}}{\mu_{0}} t^{-\mu_{0}}\right) v\right)=\exp \left(-\frac{\delta^{\prime}}{\mu_{0}} t^{-\mu_{0}}\right) \tilde{P} v
$$

that is, $\tilde{P} v=0$, where $\tilde{P}:=\exp \left(\delta^{\prime} t^{-\mu_{0}} / \mu_{0}\right) \circ P \circ \exp \left(-\delta^{\prime} t^{-\mu_{0}} / \mu_{0}\right)$. We have only to show that $v=0$ for $t>0$ in a neighborhood of $(0,0)$.

By an argument similar to and easier than that in the proof of Lemma 3.3, the 
operator $\tilde{P}$ is an operator of the form (1.1) and satisfies the following:

(a) The operator $\tilde{P}$ satisfies (A-0), (A-1), and (A-2; $\left.\mu_{0}\right)$.

(b) $S(\tilde{P}) \cap\left(\mu_{0}, \infty\right)=S(P) \cap\left(\mu_{0}, \infty\right)$.

(c) $\mathscr{C}_{\nu}[\tilde{P}](x ; \cdot)=\mathscr{C}_{\nu}[P](x ; \cdot)$ for every $\nu>\mu_{0}$ and $x \in \Omega_{0}$.

(d) $\mathscr{C}_{\mu_{0}}[\tilde{P}](x ; \lambda)=\mathscr{C}_{\mu_{0}}[P]\left(x ; \lambda+\delta^{\prime}\right)$.

(e) $S(\tilde{P}) \cap\left[0, \mu_{0}\right]=\left\{\mu_{0}\right\}$.

By (d) and the condition $\left(\mathrm{A}-6 ; \mu_{0}\right)$ for $P$, all the roots $\lambda$ of $\mathscr{C}_{\mu_{0}}[\tilde{P}](0 ; \lambda)=0$ satisfy $\operatorname{Re} \lambda<0$. This and the conditions (c), (e) imply that the operator $\tilde{P}$ satisfies $(\mathrm{A}-3)$. Further, also by $(\mathrm{d})$, we have $\mathscr{C}_{\mu_{0}}[\tilde{P}](0 ; 0) \neq 0$. This and the assumption $\left(\mathrm{A}-2 ; \mu_{0}\right)$ imply $(\mathrm{A}-2 ; 0)$. Thus, we can apply Theorem 5.1 to $\tilde{P}$, and hence, we have $v=0$ for $t>0$ in a neighborhood of $(0,0)$.

\section{REFERENCES}

[1] Baouendi, M. S. and Goulaouic, C., Cauchy problems with characteristic initial hypersurface, Comm. Pure Appl. Math. 26 (1973), 455-475.

[2] - Cauchy problems with multiple characteristics in spaces of regular distributions, Russian Math. Surveys 29 (1974), 72-78.

[3] Mandai, T., Characteristic Cauchy problems for some non-Fuchsian partial differential operators, J. Math. Soc. Japan 45 (1993), 511-545.

[4] - Distribution null-solutions for a class of non-Fuchsian partial differential operators, in preparation.

Department of Mathematics

Faculty of General Education

Gifu University

Yanagido 1-1, Gifu 501-11

Japan

E-mail address:mandai@cc.gifu-u.ac.jp 